\title{
Computed X-ray Tomography of Powder Metallurgy Product for Rapid, Quantitative Size and Shape Distribution Analysis
}

\author{
Noah Budiansky ${ }^{1}$, Daniel P. Dennies ${ }^{2}$, Joel Forman ${ }^{1}$, David Wong ${ }^{1}$, Joe Tucker $^{3}$ \\ 1. Exponent, Natick, MA. \\ 2. Exponent, Irvine, CA \\ 3. Exponent, Atlanta, GA
}

Analysis of powder metallurgy powders is an important consideration in designing the appropriate forming and processing parameters for near-net-shape components and additive manufacturing in various industries, including aerospace and electrical connectors. Not only is the size distribution an important factor in determining the packing factor of the components, but also particle shape. Typically, the particles are assumed to be spherical, with no satellites, and sparse particle analysis is conducted. Often, differences in particle size distribution, and possibly the shape of the particles, may lead to differences in the density of the finished product and overall quality. In most cases, the particle size distribution is quantified using a two-dimensional (2D) process, e.g., scanning electron microscopy (SEM) imaging or optical particle size counter or even just a mechanical sieve. Methods that would provide a more representative characterization of the particles would benefit additive manufacturing component producers in the areas of quality control, product improvement, and computational modeling.

The use of SEM with energy dispersive X-ray microscopy (EDS) for particle size measurements and elemental composition of the particles is a popular method of conducting particle characterization. The imaging mode of the SEM is used to collect representative micrographs of the particles, as shown in Figure 1. Using image analysis software to segment the particles, the 2D particle size distributions can be determined from a montage of collected images. In addition, the elemental composition of each, or a selection, of the particles can be determined using EDS. Unfortunately, this analysis can be time consuming and the number of particles evaluated may be limited and/or not representative of the entire population, specifically the extreme values. Finally, since the shape of each particle is only captured in 2D, the particle shape is likely not representative, especially in highly non-spherical particle powders.

The use of computed X-ray tomography (CT) can greatly benefit particle characterization by providing a three-dimensional (3D) analysis of each particle in a powder sample; providing a more comprehensive representation than other $2 \mathrm{D}$ methods. $\mathrm{CT}$ is a radiographic technique where a series of X-ray images are reconstructed into a 3D model of the powder sample. Using alignment, segmentation, and registration routines, each particle can be uniquely identified from the rest of the sample. In addition, a larger number of particles can be evaluated in each data acquisition, providing a more accurate representation of powder characteristics (e.g., size and shape). From simple aspect ratios to more complex moment invariants, distribution analysis of particle shape can be used to glean more detailed statistics and better inform structure-property relations.

Figure 1 and Figure 2 represents work performed on the same lot of 6Al-4V titanium powder used for additive manufacturing. Here, comparative results of the effective particle diameter for CT which analyzed approximately 131,000 particles and SEM images with approximately 271 particles indicated a distribution centered on 65 um vs 45 um, respectively. In addition, the CT analysis resulted in an improved size distribution indicating an apparent trimodal population versus a bimodal distribution from 
the SEM images. Lastly, the CT analysis indicated a higher percentage of both fines and larger particles, which indicates a less optimum powder distribution.

In summary, this work illustrates the complimentary benefit of using CT to provide a more comprehensive representation of each particle. In a matter of a few hours the CT process was able to examine almost 500x more particles from a 3D perspective than 2D SEM imaging and produced more detailed and more informative results. The results of this type of analysis can be used provide a more comprehensive quantitative characterization of the particles for quality control and product or process improvement. In addition, more accurate inputs for computational modeling that utilizes the packing behavior of specific particle size and shape distribution of a powder product can be conducted. These high fidelity models, used within an integrated computational materials engineering ("ICME") framework, stand to significantly reduce the advanced materials development and deployment cycles.
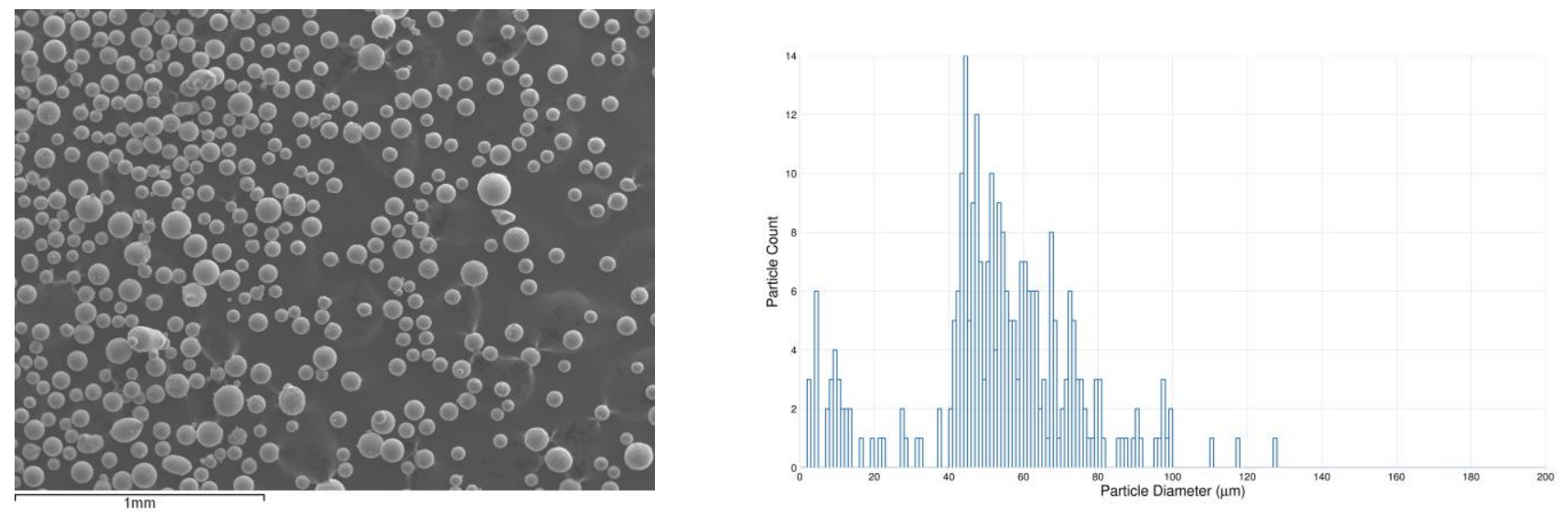

Figure 1. Scanning electron microscope image (left) and particle size distribution (right) for titanium metal powder A.
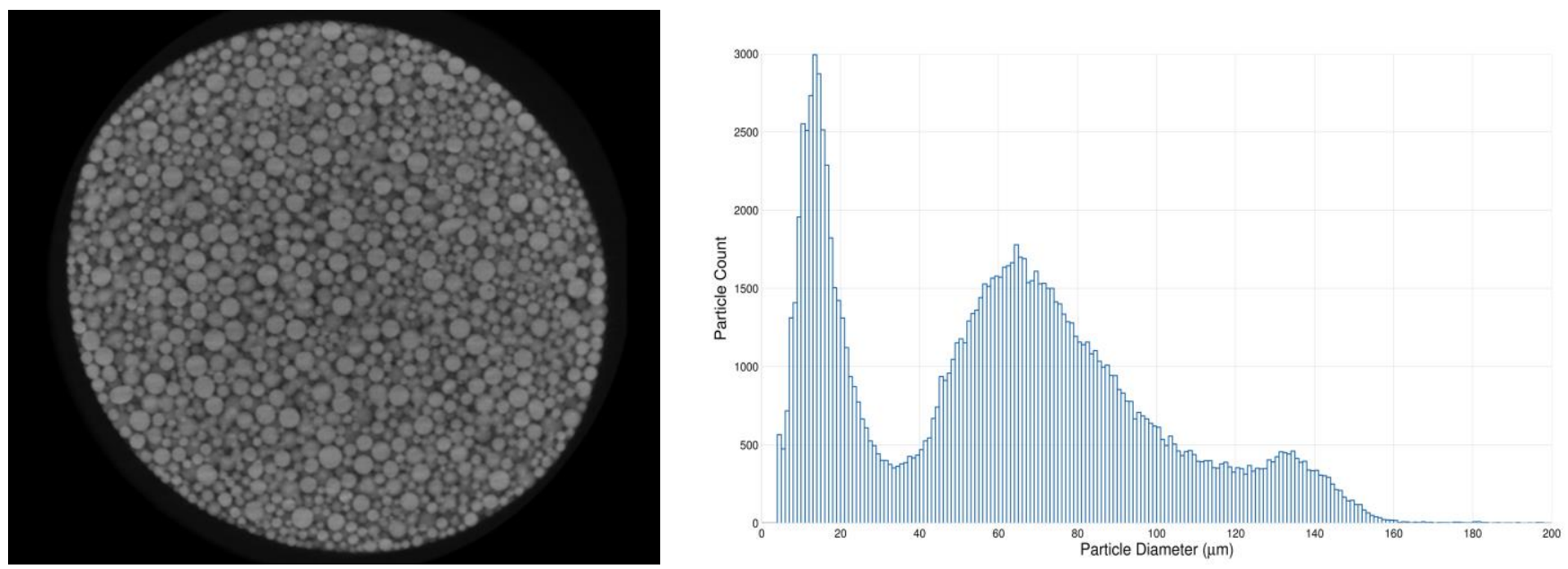

Figure 2. Computed X-ray tomography reconstruction (left) and particle size distribution (right) for titanium powder A. 